\title{
Aplasia de las válvulas pulmonares
}

\author{
Dra. Odette Farrú A.: 1 Dr. Mauricio O'Connel J.1; Dr. Ignacio Hernández N.1; \\ Sr. Rodrigo Chuaqui F. 2
}

Aplasia of the pulmonary valve

The clinical, phonocardiographic, hemodynamic and angiocardiographic findings in 8 children with aplastic pulmonary valve are presented. All had a ventriculat septal defect and in 6 there was also an infundibular pulmonic stenosis. Clinically, the most striking feature was late, low frecuency diastolic murmur of crecendo decrescendo quality and maximal intersity in the second and third left intercostal space, asociated with single, normal intensity second sound and, radiologically, abnormal parahiliar shadows, specially on the right side, or aneurysmatic dilatation of the pulmonary artery trunk. The angiocardiographic work-up usually showed aneurysmatic dilatation of the pulnonary artery trunk or his main branches and sudden peripheric reduction of their humen. These findings are not seen in any other heart discase. The ethology and results of surgical treatment of this abnormality are analized.

(Key words: Pulmonary valve, absence, aplasia, agenesia, aneurism, insuficiency, congenital yentricular septal defect, Fallot's tetralogy).

La aplasia de las válvulas pulmonares fue descrita por primera vez en 1847 por Chevers ${ }^{1}$ en un sindrome que incluía un defecto del septum interventricular (CIV), estenosis anular de la arteria pulmonar con o sin estrechamiento infundibular y dos manifestaciones adicionales únicas: regurgitación pulmonar secundaria a la ausencia de válvulas pulmonares y una dilatación aneurismática del tronco de la pulmonat y sus ramas; ochenta años después, en 1927, Kurtz ${ }^{2}$ publicó otro caso. A partir de 1954 las comunica. ciones de esta anomalía se hicieron más frecuen. tes, pero sólo en $1958^{3.4}$ se logró una correla. ción clínico-patológica, lo que permitió establecer características clínicas propias ${ }^{5-7}$.

Esta rara malformación cardíaca muy pocas veces se manifiesta como una lesión aislada: únicamente 6 casos han sido descritos ${ }^{8-13}$. Lo habitual es que se asocie a otras anomalías, más frecuentemente tetralogía de Fallot ${ }^{13-21}$ o CIV con o sin estenosis infundibular pulmonar, dando origen a un síndrome bastante característico $0^{3}, 4$, 13, 22-27. Hasta la fecha 289 casos han sido publicados $13.34,36,37$.

El objetivo de este trabajo es destacar los hallazgos clínicos en pacientes con ausencia con-

1. Undad de Cardiología. Depto. de Pediatria, Hospital Roberto del Río.

2. Interno de Medicina, Universidad Católica de Chile. génita de las válvulas pulmonares, haciendo especial énfasis en los criterios auscultatorios, fono. cardiográficos y radiológicos que deben conducir a un diagnóstico correcto.

\section{PACIENTES Y METODOS}

F.l material lo constituyen ocho niños con ausencia congénita de las valvas pulmonares, cuyas edades fluctuaron entre $21 / 2$ meses y 12 artos, 5 de sexo masculino, 3 femenino, todos con estudio clínico completo, fonocardiográfico, hemodinámico y angiográfico (en 2 casos seriado). En 3 pacientes el diagnóstico fue confirmado mediante necropsia.

\section{RESULTADOS}

Cinco pacientes eran de sexo masculino, los 8 casos tenían CIV, en 6 habia, además, estenosis pulmonar infundibular (3 leve, 3 moderada) y en uno de ellos estenosis pulmonar anular; en otro caso además de la CIV y la estenosis infundibular había una comunicación interauricular (CIA). Cuatro de los 8 casos tenian el arco aórtico a derecha y 4 agenesia de la rama izquierda de la arteria pulmonar; dos pacientes eran hermanos.

La cianosis era poco intensa, por lo general leve o moderada, con grados variables de retardo del desarrollo pondo-estatural y limitación de la capacidad funcional. Dos pacientes fallecieron durante el intento de corrección quirúrgica y un tercero a los $21 / 2$ meses en insuficiencia cardiaca refractaria (Fig. 1). 


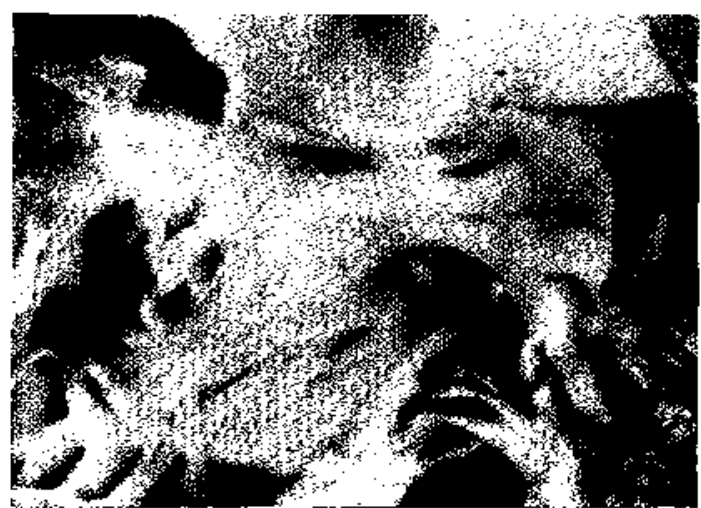

Figura 1: Arlasia de válvulas pulmonares. Tracto de salida del ventriculo derecho y porción inicial del tronco de la arteria pulmonar. Anillo pulmonar hipoplásico y sin evidencia de estructura valvular. Dilatación del tronco pulmonar e hipertrofia ventricular derecha.

Los hallazgos electrocardiográficos fueron notablemente uniformes en todos los casos con importante sobrecarga de cavidades derechas, y bloqueo, incompleto o completo, de rama dere. cha.

Auscultatoriamente y fonocardiográficamente (Fig. 2 y 3) todos los niños afectados tenian soplos holosistólicos de variable configuración (todos tenían cortocircuitos de izquierda a derecha), el segundo ruido invariablemente era único y no acentuado. En ningún caso existía clic sistólico. En todos había un soplo diastólico tardio, creciente-decreciente, de baja a mediana frecuencia, en toda la extensión del borde izquierdo esternal, pero especialmente en el tercer espacio intercostal izquierdo.

En el estudio radiológico el indice cardiotorácico fue mayor de 0,5 en cada caso. Lo más llamativo fue (Fig. 3 y 4 ) la existencia de grandes sombras patológicas parahiliares. especialmente a derecha o ensanchamiento bilateral del pediculo vascular o dilatación acentuada del tronco de la arteria pulmonar, con circulación perférica normal o disminuida. Franca oligoemia izquierda en
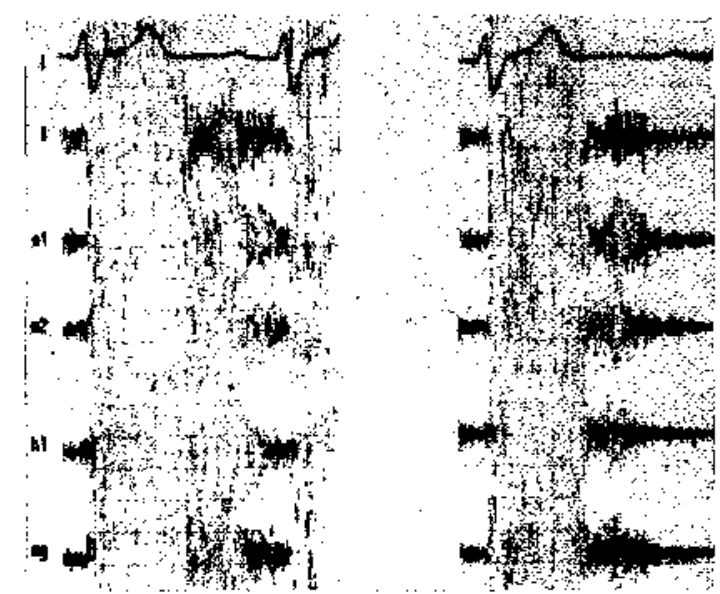

Figura 2: Aplasia de vábulas pulmonares. Fonocardiograma: a la izquierda, en tercer espacio intercostal izquierdo (con diferentes tiltros), a la derecha, en el apex cardíaco. Soplo holosistólico intenso de alta frecuencia, creciente-decreciente precoz, más intenso en tercer espacio intercostal izqujerdo. $2^{\circ}$ ruido englobado por el soplo sistólico, no acentuado.

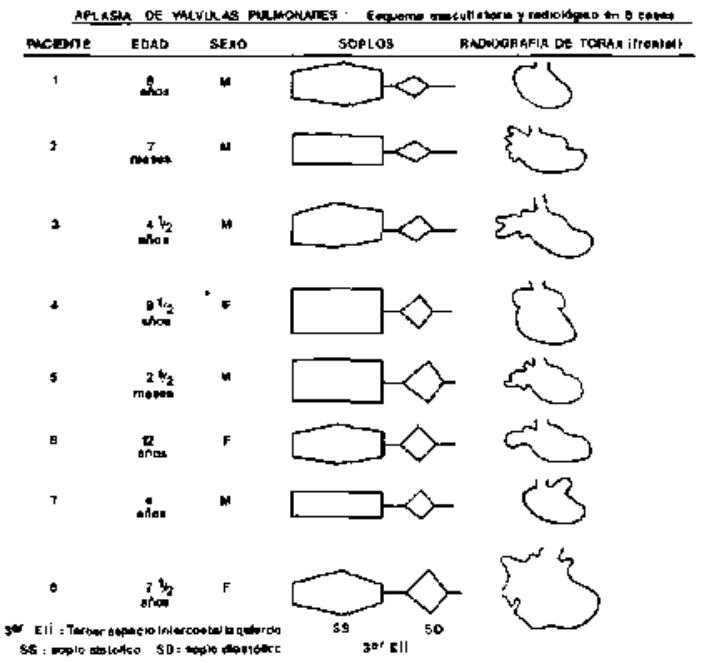

Figura 3: Aplasia de válvulas pulmonares. Esquema auscultatorio y radiológico. Véase texto.
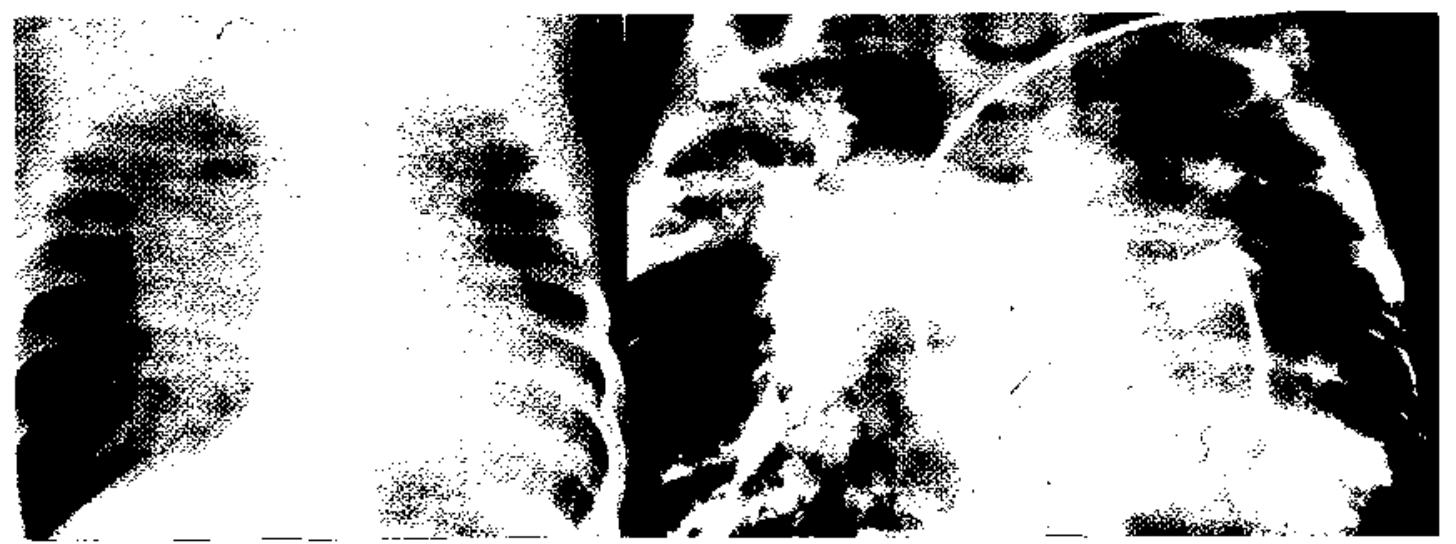

Figura 4. Aplasia de válvulas pulmonares. Aspec tos radiológicos y angiocardiográfícos. 
los casos con agenesia de rama izquierda.

El estudio hemodinámico reveló en todos los pacientes presiones en el ventrículo derecho (VD) iguales o cercanas a las sistémicas, presión elevada en aurícula derecha (AD), en varios casos con onda "a" prominente. Sólo en un paciente la presión diastólica en arteria pulmonar (AP) era baja. Existían gradientes sistólicos variables en el infundibulo del ventrículo derecho, excepto en un caso que no tenía estenosis infundibular y en otro cuya necropsia mostró una estenosis infundibular leve. Sólo en 2 pacientes había franca insaturación arterial de oxígeno, en 3 era leve $y$ en los otros que la saturación era normal. Todos tenian evidencia de cortocircuito de izquierda a derecha a nivel ventricular y 2 de derecha a izquierda en el mismo nivel y detectable en reposo.

E] hecho angiocardiográfico más importante fue el aspecto de los vasos pulmonares principales con dilataciones aneurismáticas ya sea del tronco de la AP, rama derecha o izquierda (Fig. 4), siendo lo más frecuente la dilatación de la rama derecha de la AP ( 7 casos). La válvula pulmonar no se visualizó nunca, pero en $4 \operatorname{casos}^{2}, 3,5,7$ existía un estrechamiento diafragmático en la posición de la válvula pulmonar, sin cúpula ni chorro de medio de contraste.

Tan llamativo como la dilatación de los vasos pulmonares principales fue el cambio brusco de calibre entre ellos y sus ramas de segundo orden, las que eran comparativamente estrechas. En 3 casos no existía rama izquierda, en ellos había oligoemia en el pulmón izquierdo.

\section{DISCUSION}

La aplasia de las válvulas pulmonares generalmente se asocia a tetralogía de Fallot o CIV $(92 \%)$ y frecuentemente se agrega una estenosis pulmonar infundibular, que protegería al ventrículo derecho (VD) de las consecuencias hemo. dinámicas de la ausencia de las válvulas. Habitualmente la estenosis pulmonar infundibular es leve - moderada y por esto rara vez existe cianosis marcada, dedos hipocráticos o crisis anoxémicas. La incidencia de ausencia de válvulas pulmonares en series de casos de tetralogía de Fallot varía de $2,4 \% 38$ a $6,3 \% 24$.

Unicamente se han descrito 6 pacientes $(2 \%)$ con ausencia de válvulas pulnonares sin asociación con otra malformaciốn cardiaca $a^{8,13}$. También han sido descritas otras asociaciones poco frecuentes, con anomalia de Uhl, un caso ${ }^{39}$; doble arco aórtico ${ }^{34}$, hipoplasia del VD, un $\operatorname{caso}^{40}$, doble salida del VD, tres $\operatorname{casos}^{23.30 .41}$. CIA tipo ostium secundum, cuatro casos sin incluir el nuestro ${ }^{34}, 42,43$, dextrocardia con situs inversus, un caso ${ }^{13}$ y atresia tricuspídea, en 5 pacientes 44,45 . E] septum interventricular estaba intacto sólo en 9 de 289 pacientes descritos hasta la fecha.

Los hallazgos clinicos presentados y los pu. blicados por otros autores, sugieren que la combinación de soplo pansistólico paraesternal, segundo ruido único no acentuado y un soplo diastólico (SD) tardío, creciente-decreciente, de baja frecuencia a lo largo del borde esternal izquierdo es patognómico de CIV con agenesia de válvulas pulmonares $15,31,37,46-49$.

El SD difiere del que se oye en la incompetencia pulmonar aislada (sin hipertensión pulmonar) porque comienza más tarde: Bouvaros y Ducher (1961) encontraron que el SD de la insuficiencia pulmonar aislada comienza 0,02 a $0,03 \mathrm{seg}$. de A2 mientras que en los casos de agenesia, lo hace, en promedio, 0,10 seg. después de A2. Este retardo refleja la prolongación adicional del periodo de eyección del VD debido al cortocircuito ventricular de izquierda a derecha.

Aunque la dilatación de la AP puede producir un $\mathrm{Clic}^{47}$ en ninguno de nuestros casos se registró este ruido agregado, lo que ha sido también señalado por otros autores y apoyaria a quienes sostienen que los clic son de origen valvular y no vascular y que en ausencia de válvulas, éstos no se producirian:

Mediante fonocardiografía intracavitaria se ha demostrado que los soplos diastólicos se captan más intensamente en el infundíbulo del ventrículo derecho 46,47 (insuficiencia pulmonar).

En el electrocardiograma la existencia de bloqueo completo o incompleto de rama derecha, es un elemento valioso para diferenciar la aplasia de las vályulas pulmonares de la tetralogia de Fallot.

Las radiografías de tórax generalmente muestran cardiomegalia moderada, pero también han sido descritos casos con silueta cardíaca de tamaño normal. La cardiomegalia ha sido relacionada con la severidad de la regurgitación pulmonar ${ }^{37}$. La dilatación de la arteria pulmonar se puede observar claramente en la proyección frontal, y la arteria pulmonar izquierda, puede identificarse más fácilmente en la proyección oblicua izquierda anterior. La causa de la dilatación de la arteria pulmonar es aún incierta; pero se cree que resulta del aumento de volumen del VD $3 \%$ 50. 1 causado por la regurgitación pulmonar y el cortocircuito de izquierda a derecha a través del defecto interventricular. Otros autores sostienen que se dẹbe a alteraciones estructurales del tejido elástico de $\operatorname{los} v a s o s^{31}$. La dilatación de la arteria puimonar y sus ramas contrasta con la vascularización pulmonar periférica, que generalmente está disminuida. En los casos en que existe 
agenesia de una de las ramas se observan netas diferencias en el número y el calibre de los vasos entre ambos pulmones.

Aunque el diagnóstico de aplasia de las válvulas pulmonares puede hacerse fácilmente con los signos auscultatorios y radiológicos, el estudio ecocardiográfico puede ser de mucha ayu$\mathrm{da}^{52.54}$. Con este método pueden observarse signos de sobrecarga de volumen del VD, dilatación aneurismática de la arteria pulmonar y cabalgamiento de la aorta. Otros hallazgos, también descritos, son la obstrucción pulmonar, la ausencia de ecos de las vălvulas o la presencia de otros originados en las válvulas rudimentarias.

El cateterismo cardíaco es importante para identificar el sitio de la obstrucción del tracto de salida del VD, que generalmente se localiza en el anillo valvular ${ }^{16}$. En algunos casos sin embargo se han encontrado obstrucciones anulares e in. fundibulares ${ }^{15.31}$.

Los hallazgos angiocardiográficos son muy llamativos: no hemos visto en ninguna otra condición dilataciones tan grandes del tronco de la AP y sus ramas, ni tampoco la brusca transición de calibre entre los troncos pulmonares y sus ramas, como ocurren en esta anomalia.

El pronóstico de estos pacientes depende de la edad de aparición y severidad de los síntomas y de la presencia y grado de regurgitación pulmonar $13,20,34,35,50$. En los lactantes, principalmente en los menores de un año, los resultados del tratamiento quirúrgico son desalentadores, asociándose a alta mortalidad $(76-94 \%)^{13}, 15$. 16. 50 por lo que muchos autores recomiendan 34,5051 un tratamiento médico vigoroso con el propósito de mejorar sus condiciones generales e intentar la operación correctora a mayor edad.

En los niños mayores en cambio, se recomienda cerrar la CIV y resecar el tracto de salida del VD. En los pacientes en quienes se efectúa además dilatación del anillo pulmonar sin implantar prótesis, se ha observado masiva regurgitación con aumento de la presión sistólica de la arteria pulmonar. Seguimientos posteriores han demostrado que su evolución ha sido similar a la observada en niños no operados, en quienes la insuficiencia pulmonar produce una dilatación progresiva de la arteria pulmonar con compresión bronquial, enfisema, dificultad respiratoria $y$ muerte.

La colocación de una prótesis valvular en el anillo pulmonar durante la operación inicial continúa siendo motivo de discusión y controversia porque se calcifican y fallan precozmente en los pacientes jóvenes $20,33-35,43$. Sin em. bargo, a pesar de los inconvenientes, hay quienes consideran que corregir la malformación utili- zando prótesis en la operación inicial puede preservar la contractilidad y funcionalidad del VD al impedir la regurgitación pulmonar 5,13 , $20,34,48$.

\section{RESUMEN}

Se presentan los hallazgos clínicos, fonocardiográficos, hemodinámicos y angiocardiográficos- de 8 niños portadores de una aplasia de válvulas pulmonares. Todos con CIV asociada, 6 con estenosis pulmonar infundibular. Clínicamente destaca la existencia de un segundo rujdo único no acentuado, un soplo diastólico tardio creciente-decreciente en el 20 y 3 er espacio intercostal izquierdo junto a una imagen radiológica con sombras patológicas parahiliares, especialmente a turecha o gran dilatación del tronco de arteria pulmonar. Los hallazgos angiográficos son muy llamativos, no existiendo en ninguna otra patología dilataciones tan groseras del tronco de la arteria pulmonar o sus ramas, así como la brusca transición de calibre entre los troncos pulmonares principales y sus ramas. Se discute la etiologia de esta patología y se analizan los resultados quirúrgicos.

\section{REFERENCIAS}

1. Chevers. $N_{.:}$Recherches sur les maladies de l'artére pulmonaire. Arch. Gen. Med. $15 \div 488,1847$.

2. Kurz, C.M,; Sprague, H.B.: White. P.D.: Congenital heart disease: Intraventricular septal defect with associated anomalies in serie of three cases examinated post mortem and living patient fifty eight year old with cyanosis and clubbing of fingers. Am. Heart. J. 3: 77,1927

3. Milier, R.A.; White. H, Lev, M.: Congenital absence of the pulmonary valve. Clinical and pathological syndrome. Circulation (Abstr) 18: 759, 1958.

4. Onesti, S.J., Harned, H.S.: Absence of the pulmonary valve associated with renticular septal defect. Am. J. Catdiol. 2: 496, 1958.

5. Lakier, J.B.; Stanger, P.: Heymann, M.A. et al.: Tetralogy of Fallot with absent pulmonary valve: natural history and hemodynamic considerations. Circulation 50: 167, 1974.

6. Emmanoutlides, G.C.: Congenjtal absence of the pulmonary valve. IN: Moss A.J.: Adams F.H.; Emmanoulidies G.C. (ed.): Heart Disease in Infants, Children and Adolescents. $2^{\text {a }}$ ed. Baltimore: Williams and Wílkins pp. 258, 1977.

7. Howkins, J.A.; Khoury, G.H.; Batriste, C.E.; Byme, J.P.: Tetralogy of Fallot: cases with absent pulmonary valve, J. Kans. Med. Soc. 81:400, 1980.

8. Ehrenhaft, J.L.: Discussion on experimental pulmonary insufficiency. I. Thorac Cardiovasc. Surg. 30: $633,1955$.

9. Ho, T.: Engle, M.A.; Holswade, G.R.: Congenital insufficiency of the pulmonic valve; a rare cause of neonatal heart failure. Pediatriccs 28: 712, 1961.

10. Osman, M.A.: Meng, C.C.L.; Girdnay, B.R.: Congenital absence of the pulmonary valve; Ieport of ejght cases with review of the literature. Am. J. Roentgenol 106: 58, 1969. 
11. Pouget, J.M.; Kelly, C.E.; Pliz, C.G.: Congenital absence of the pulmonic valve: report of a case in a 73 year old man Am. J. Cardiol. 19: 732, 1967.

12. Smith, R.D.; DuShone, J.W.; Edwards, J.E.: Congenital insufficiency of the pulmonary valve. Including a case of fetal cardiac failure. Circulation 20 : $554,1959$.

13. Calder, A.L.; Brandt, P.W.T.; Barrat-Boyes, B.G. Netze, J.M.: Variant of Tetralogy of Fallot with absent pulmonary valve leaflets and origin of one pulmonary artery from de ascending aorta. J. Cardiol. 46: 106, 1980.

14. Rowe, R.D.; Vlod, P.; Keith, J.D.: Atypical tetralogy of Fallot A noncyanotic form with increased lung vascularity. Circulation 12:230, 1955 .

15. Miller, R.A.; Lev, M.i Poul, M.H.: Congenital absence of pulmonary valve. The clinical syndrome of tetralogy of Fallot with pulmonary regurgitation. Circulation 26: 266, 1962.

16. Ruttenberg, H.D.; Corey, L.S.; Adams, P. Jr.; Edwords, J.E.: Absence of the pulmonary valve in the Tetralogy of Fallot. Am. J. Roentgenol $91: 500$, 1964 .

17. Nagao, G.I.; Daoud, G.I.; McAdams, A.J. ef al.: Cardiovascular anomalies associated with tetralogy of Fallot. Am. J. Cardiol. 20: 206, 1967.

18. Bove, E.L.; Shaher, R.M.; Alley, R.M.; McNeally, $M$.: Tetralogy of Fallot with absent pulmonary valve and aneurysm of the pulmonary artery. Report of two cases presenting as obstructive lung disease. J. Pediatr. 81: 339, 1972.

19. Emmanouilides, G.C. Thanopoulos, B.; Siassi, B.; Fishbein, $M_{\text {.: }}$ Agenesis of ductus arteriosus associated with the syndrome of Fallot and absent pulmonary valve Am. J. Cardiol. 37: 403, 1976.

20. Pinsky, W.W.; Nihill, M.R.; Mullins, C.E.; Harrison, $G$, McNamara, D.G.: The absent pulmonary valve syndrome: considerations of management. Circulation 57: 159, 1978.

21. Zach, M.; Beitzke, A.; Singer, H.; Hofler, H.; Schellmann, B.: The syndrome of absent pulmonary valve and ventricular septal defect: anatomical features and embryologic considerations Basic Res. Cardiol. 74: 54, 1979

22. Soulie, P.; Vermont, P.; Sterbar, S. ef al.: Insuffjcience pulmonaire congenital et communication interventticulaire. Arch. Mal Coeur, 60: 172, 1967.

23. Durnin, R.E.; Wilner, R.; Vinmani, S.; Laurence, $T$.; Fyles, D.C.: Pulmonary regurgitation with ventricular septal defect and pulmonary stenosis-tetralogy of Fallot variant. Am. J. Roentgenol 106: 42, 1969.

24. Lev, M.; Eckner, F.A.O.: The pathologic anatomy of tetralogy of Fallot and its variations. Dis. Chest. 45: 2\$1, 1964.

25. Campeu. LA.; Ruble, P.E.; Cooksey, W.B.: Congenital absence of the pulmonary valve. Circulation $15: 397,1956$

26. Campeau. L.; Gilbert G.: A erichide, $N$.: Absence of the pulmonary valve. Report of two cases associated with other congenital lesions. Am. J. Cardiol. 8: $113,1961$.

27. Venabler, $A . W .:$ Absence of the pulmonary valve with ventricular septal defect. Br. Heart. J. 24: 293, 1962 .

28. Elliot, L.P,; Shanklin, D.R,; Schiebler, G.L.: Congenital insufficiency of the pulmonary valve with il ventricular septal defect. Dis. Chest. $42: 534,1962$.

29. Childers, R.W.; McCred. P.C.: Absence of the pulmonary valve A case ocurring in the Marfan syndrome. Circulation 29: $598,1964$.
30. D'Cruz, I.A.; Lendrum, B.L.; Novak, G.: Congenital absence of the pulmonary valve. Am. Heart. J. 68 : 728,1964 .

31. Macartney, F.J.; Millet, GA.H.: Congenital Absence of the pulmonary valve. Br. Heart. J. 32: 483, 1970.

32. Porker, M.E.; ABT, A.B.; Porr, G.V.S.; Fallot's Tetralogy: its occurrence with absent pulmonary valve and sinus of valsalva aneurysm in an adult. Arch. Pathol Lab. Med. 104: 597, 1980.

33. Stafford, E.G.; Mair, D.D.; MCGoon, D.C.; Damielson, $G$. $K$.: Tetralogy of fallot with absent pulmonary valve: surgical considerantions and results. Circulation 47, 48: suppl.. (III): III. 24, 1973.

34. Ibawi, M.N.; Idriss, F.S., Muster. A.J. y cols.: Tetualogy of fallot with atsent pulmonary valve. J. Thorac. Cardiovasc. Surg. 81: 906, 1981.

35. Dunnigan, A.; Otdham, H.N.; Benson, D.W.: Absent Pulmonary Valve Syndrome in infancy: Surgery Reconsidered. Am. J. Cardiol. 48: 117, 1981.

36. Hueb, W.A.; Mazzien, R.: Oliveira, H.A.; Sowza, JM.; Palácios, E. Oliveira, S.A.: Agenesia da valve pulmonar asociada a comunicacáo interventricular. Arq. Bras Cardiol. 36: 53, 1981.

37. Buendia, A.; Attie, F.; Ovseyevitz y cols.: Congenital absence of pulmonary valve leaflets. Br. Heart J. $50: 31,1983$.

38. Rao. B.; Anderson, R.; Edwards, J.E.: Anatomic Variations in the Tetralogy of Fallot. Am. Heart. J. 81: $361,1971$.

39. Koul, M.; Arora, $R$; Ram, $S$.: Uhl's anomaly with rudimentary putmonary value leaflets. A clinical, hemodynamic; angiographic and pathologic study. Am. Heart. J, 100: 673, 1980.

40. Franco Vásquez, I.S.: Riojas Dávila. U.; Angulo, H.O.: Pérez Trevino, C.: Ausencia congénita de la válvula pulmonar con hipoplasia del ventrículo derecho. Presentación de un caso. Arch. Inst. Cardiol Mex. 39: 865, 1969.

41. Sridamont, S.; Feldt, R.H.; Ritter, D.G.: Davis, G.D.; McGoon, D.C., Edwards, J.E.: Double-outlet right ventricle associated with persistent common atrioventricular canal. Cículation 52: 933, 1975.

42. Chiemmong Koltip, P.: Replogle, R.L.; González-Lavin, L.; Arcilla, R.A.: Congenital absence of the pulmonary yalve with atrial septal defect surgically corrected with aortic homograft. Chest. 62: 100,1972 .

43. Zajtchuk, R.: González-Lavin, L.; Replogle. R.L.: Pulmonary artery aneurysm associated with atrial septal defect and absent pulmonary valve. J. Thorac. Cardiovasc. Surg. 65: 699, 1973.

44. Marin-Garcia, J.; Roca, J.; Blieden, L.C.; Lucas, R.V. Jr.; Edwards, J.E.: Congenital absence of the pulmonary valve associated with tricuspid atresia and intact ventricular septum. Chest. 64: 658, 1973.

45. Quero Jimenez, M.: Maitre Azcarate, Mt.J.; Alvarez Mejarano, $H_{1}$; Vasquez Martel, E.: Tricuspid atresics. An anatomical study of 17 cases. Eur. J. Cardiol. 3/4: 337, 1975.

46. Harris, B.C.; Shaver, J.A.; Kroetz, F.W.; Leonard, I.J.: Congenital pulmonary valvular insufficiency complicating tetralogy of fillot. Intracardiac sound and pressure correlates. Am. J. Cardiol. 23: 864, 1969.

47. Pérez Trevino, C.; Wabi Droge, C.: Agenesia de las válvulas pulmonares. Presentación de l1 casos y revisión de literatura. Arch. Inst. Cardiol. Méx. 42: 33,1972 . 
48. Cox, JN., de Seignewx, R.; Bolens, M,; Haenni, $P$. Bopp, P.; Bruins, C.: Tricuspid atresia, hypoplastic right ventricle, intact ventricular septum and congenital absence of the pulmonary valve. Helv. Paediatr. Acta 30: 389, 1975.

49. Fontana, M.E.: Wooley, C.F.: The murmur of pulmonic regigitation in tetralogy of Fallot with absent pulmonic valve. Circulation $64: 558,1978$.

50. Byrne, J.P.; Hawkins, J.A.; Battiste, C.E.: Khoury, G.H.: Palliative Procedures in Tetralogy of Fallot with Absent Pulmonary Valve: A new Approach. Ann. Thorac. Surg. 33: 499, 1982.

51. Arensman, F.W.; Francis, P.D.; Helmsworth, J-A+; Benzing III G.; Schreiberg, J.T.; Schwartz, D.C.; Kaplon, S.: Early medical and surgical intervention for tetralogy of Fallot with absence of pubnonic valve. J. Thorac. Cardiovasc. Surg. 84: 430, 1982.

52. Weyman, A E.; Dillon, J.C.; Feigenbaum, H.; Chang, $S$.: Pulmonary valve echo motion in pulmonary J egurgitation. Br. Heart. J. 37: 1184, 1975.

53. Chedtham, J.P.; Latson, LA.; Gutgesell, H.P.; Echocardiographic pulsed Doppler features of absent pulmonary valve syndrome in the neonate. Am. J. Cardiol. 49: 1773, 1982.

54. Segni, E.D.; Einzlg, S.: Boss, J.L.; Edwards, J.. Congenital Absence of Pulnonary Valve Associated with Tetralogy of Fallot: Diagnosis by 2 - Dimen. sional Echocardiography. Am. J. Cardiol. 51: 1798, 1983. 